\title{
Eficácia da crioimersão e massagem desportiva na recuperação de atletas: uma revisão da literatura
}

\section{Efficacy of cryoimmersion and sports massage in the recovery of athletes: a review of the}

\author{
literature.
}

\section{Lucas Sousa Rocha 1; Rodrigo Disconzi Nunes 2; Geovane Rossone Reis ${ }^{3}$; Edison Benedito da Luz Brito Junior ${ }^{4}$}

\section{RESUMO}

Introdução: A fadiga muscular é o sintoma mais comum após exercícios extenuantes, ocorrendo dano muscular por indução de uma resposta inflamatória. Com a finalidade de diminuir os sinais de inflamação gerados pelo exercício, intervenções terapêuticas são realizadas. A imersão em água fria e massagem desportiva são comumente utilizadas. Objetivo: Verificar a eficácia da técnica de crioimersão e massagem desportiva na recuperação muscular de atletas pós-exercícios. Material e Método: Trata-se de uma revisão bibliográfica de literatura. A busca dos artigos foi realizada através das bases de dados eletrônicos, sendo incluídos estudos que abordaram a utilização da crioterapia e massagem desportiva como técnicas de recuperação muscular pós-exercício físico. Resultados: Foram selecionados 20 artigos, demonstrando eficácia das duas técnicas, diminuindo principalmente a percepção de dor e fadiga. Conclusão: Crioimersão e Massagem desportiva parecem ser duas técnicas eficazes na recuperação de atletas pós-exercício físico, podendo ser aliados dos fisioterapeutas esportivos na prevenção e reabilitação de atletas.

Palavras-chave: Crioterapia. Massagem. Fisioterapia.

\section{ABSTRACT}

Introduction: A pain and muscle fatigue are the most common symptoms after strenuous exercise, muscle damage occurring by induction of an inflammatory response. In order to reduce the signs of inflammation generated by the exercise, therapeutic interventions are performed. Cold water immersion and sports massage are commonly used. Objective: To verify the effectiveness of the technique of cryoimmersion and sports massage in the muscular recovery of athletes after exercises. Method: This is a bibliographical review of literature. The articles search was carried out through the electronic databases, and studies were included that addressed the use of cryotherapy and sports massage as techniques of muscle recovery after physical exercise. Results: Twenty articles were selected, demonstrating the effectiveness of both techniques, mainly reducing the perception of pain and fatigue. Conclusion: Cryoimmersion and Sports Massage seems to be two effective techniques in the recovery of athletes after physical exercise, being able to be allies of Sports Physiotherapists in the prevention and rehabilitation of athletes.

Keywords: Cryotherapy. Massage. Physiotherapy
Fisioterapeuta graduado na Universidade de Gurupi- UnirG. Av. Rio de Janeiro, centro, Gurupi-TO, Brasil.

E-mail:

lucassousafisio@gmail.com

2 Mestre em Ciências da Saúde pela Universidade Federal de Goiás - Goiânia (GO). Professor Adjunto I da Universidade de Gurupi- Gurupi-TO, Brasil.

3 Professor da Universidade de Gurupi- Gurupi-TO, Brasil.

Acadêmico do curso de medicina da Universidade de Gurupi- Gurupi-TO, Brasil. 


\section{INTRODUÇAO}

A dor e a fadiga muscular são os sintomas mais comuns após exercícios extenuantes, ocorrendo dano muscular por indução de uma resposta inflamatória e redução das reservas de energia. ${ }^{1} \mathrm{~A}$ fadiga é produto do estresse posto aos sistemas fisiológicos, e está relacionada a vários fatores extrínsecos e intrínsecos, entre eles a desidratação, diminuição do glicogênio, lesão musculoesquelética e cansaço mental, ${ }^{2}$ dificultando assim o processo de recuperação muscular, sendo que os membros inferiores são as partes do corpo que mais são atingidos pela dor e fadiga. ${ }^{3-4}$

A fim de melhorar o desempenho do atleta, a fadiga e dor muscular devem ser diminuídas através da recuperação, que restaura o processo fisiológico e psicológico do corpo. ${ }^{5}$ Sendo assim, a recuperação dos atletas ganhou maior credibilidade nos últimos anos, onde Fisioterapeutas Esportivos utilizam com frequência protocolos recuperativos pós exercícios ${ }^{6}$ com o objetivo de minimizar a fadiga após o exercício, prevenir a dor muscular de início tardio (DOMS) e diminuir os riscos de lesões, ${ }^{7}$ pois o melhor desempenho esportivo do atleta está diretamente ligado com a maior rapidez na recuperação. $^{8}$

Com a finalidade de diminuir os sintomas causados pós-exercícios exaustivos, intervenções terapêuticas são realizadas. A massagem desportiva é uma das condutas mais utilizadas, definida como uma técnica de compressão manual e percussões rítmicas do tecido muscular visa aumentar o fluxo sanguíneo e linfático, eliminando os catabólitos, reduzindo assim a sensação de fadiga e alívio da dor. A massoterapia baseia-se fisiologicamente pela liberação de $\beta$-endorfinas e o efeito neurológico, causado pelo estímulo mecânico através do contato manual na pele. ${ }^{9}$

Outra estratégia comum de recuperação em atletas é a imersão em água fria, com o objetivo de diminuir o início tardio de dor muscular e reduzir a percepção de fadiga, ${ }^{10}$ baseando-se pela fisiologia da vasoconstrição periférica, reduzindo assim o processo inflamatório pela diminuição do metabolismo celular. ${ }^{11}$

O objetivo deste estudo foi verificar a eficácia da técnica de crioimersão e massagem desportiva na recuperação muscular de atletas pós-exercícios.

\section{MATERIAIS E METODOS}

O presente estudo trata-se de uma revisão de literatura e a busca dos artigos foi realizada através das bases de dados eletrônicos: Scientific Electronic Library Online 
(SCIELO), Biblioteca Virtual em Saúde (BVS) e Medical Literature Analysis and Retrieval System Online (MEDLINE). Os descritores utilizados foram: Crioterapia e massagem desportiva, fisioterapia.

Os artigos foram pré-selecionados por meio dos títulos e análise dos resumos, sendo pesquisados artigos de qualquer idioma, que foram publicados entre os anos de 2010 a 2017.

Os critérios de inclusão foram: estudos que abordaram a utilização da crioterapia e massagem desportiva como técnicas de recuperação muscular pós-exercício físico em atletas. Foram incluídas literaturas de pesquisa original, artigo de revisão e metaanálise, e excluídas literaturas com metodologias falhas e publicações que não estivessem de acordo com os objetivos da revisão.

\section{RESULTADOS}

Foram encontrados 57 artigos sobre o tema da revisão, e após a análise de dois avaliadores, foram selecionados 20 artigos que se enquadravam nos critérios para a elaboração da tabela de resultados, nos quais ofereceram conclusões significativas que estavam de acordo com o objetivo desta revisão.

Tabela 1. Autores, ano de publicação, objetivo e resultado dos artigos incluídos nesta revisão.

Autor

Crowther et al. 2017

Lombardi et al. 2017

\section{Objetivo}

Investigar as técnicas de recuperação utilizadas pelos atletas de esporte de equipe em vários níveis de competição.

Fornecer uma visão geral completa e atualizada da técnica de crioimersão.

\section{Resultado}

Crioimersão foi à segunda estratégia de recuperação mais eficaz, utilizada pelos atletas, por reduzir o edema, inflamação e diminuir a dor muscular, sendo que a primeira estratégia foi a associação entre o sono e a massagem.

Crioimersão vêm sendo demonstrada como uma estratégia preventiva contra os efeitos da inflamação induzida pelo exercício, melhorando a dor e estresse muscular, quando comparada com grupo não tratado. 
Yeung et al. 2016

Machado et al. 2016

Nunes et al. 2016

Resnick 2016

Hayter et al. 2016

Roberts et al. 2015

Hohenauer et al. 2015
Investigar os efeitos da imersão na água fria sobre a oxigenação muscular e o desempenho durante exercícios repetidos de exercício fatigante em um grupo de jovens adultos saudáveis.

Determinar a eficácia da crioimersão no manejo da dor muscular em comparação com a recuperação passiva.

Se a massagem terapêutica poderia otimizar recuperação de atletas depois de competir um triathlon de longa distância.

Comparar os efeitos da massagem e recuperação em repouso após exercício de esteira submáxima.

Comparar o efeito do crioimersão e do tratamento do ar frio sobre o desempenho máximo do ciclismo e os marcadores de dano muscular pós-exercício.

Examinar os efeitos da imersão em água fria sobre a dinâmica hemodinâmica cardíaca, termorregulação.

Massagem imediatamente após o exercício diminuiu a frequência cardíaca e teve um efeito positivo na resposta de relaxamento, comparando com a recuperação passiva.

A crioimersão minimiza os efeitos da fadiga muscular, que são induzidas pelos treinamentos de alta intensidade.

Imersão em água fria diminuiu a hemodinâmica, temperatura tecidual e manteve a força muscular. Enquanto a recuperação ativa manteve a hemodinâmica, temperatura tecidual e diminuiu a força muscular pós-exercício de resistência.

A crioimersão obteve melhor resultado, comparando com outras técnicas de resfriamento, reduzindo os 
Bervoets et al 2015

Tiidus 2015

Leeder et al. 2015

Versey et al. 2013

Delextra et al. 2012

Pointon e Duffield 2012

Crane et al. 2012 estratégias pós-exercício passivas por meio de características muito diversas após vários protocolos de exercício exaustivos.

Verificar se a massagem terapêutica é eficaz para pessoas com problemas musculoesqueléticos em relação a qualquer outro tratamento ou nenhum tratamento?

Comparar os efeitos da massagem como forma de recuperação

Verificar a eficácia das imersões em água fria sentada e em pé, na recuperação da atividade física extenuante.

Analisar os protocolos de recuperação de imersão em água investigados na literatura, seus efeitos sobre a recuperação do desempenho.

Comparar os efeitos massagem desportiva e crioimersão intermitente nos marcadores perceptivos e de desempenho da recuperação por parte dos jogadores de basquetebol após partidas competitivas.

Examinar os efeitos da crioimersão sobre a recuperação de funções neuromusculares, fisiológicas e perceptivas após o exercício simulado de esporte de equipe baseado em colisão.

Avaliar a influência da massagem no músculo que sofreu um exercício intenso, a fim de entender melhor se a massagem é uma terapia apropriada para reabilitação tecidual. sintomas de dor de início tardio e percepção de esforço.

A massagem terapêutica diminui a dor imediatamente após a intervenção e melhora a função.

Massagem teve um efeito positivo na recuperação pósexercício submáximo

É uma estratégia eficaz na diminuição da dor muscular de início tardio.

Que a imersão em água fria usada apropriadamente após o exercício, pode auxiliar na recuperação do desempenho esportivo.

Imersão em água fria e massagem desportiva tiveram uma melhora na fadiga geral e dor nas pernas. Porém a dor foi menor no grupo de crioterapia.

Crioimersão melhorou a percepção de dor pósexercício, porém não foi eficaz na redução do aparecimento de marcadores sanguíneos de dano muscular, em exercício de contato.

A massagem terapêutica parece ser benéfica, por reduzir a inflamação e promover a biogênese mitocondrial. 
Prado et al. 2012

Ascenção et al. 2011

Rowsell et al. 2011

Wiltshire et al. 2010
Analisar a influência da Crioimersão imediata ao esforço físico agudo no Estresse Oxidativo no plasma sanguíneo

Avaliar os efeitos de uma única sessão de imersão em água fria ou termo neutral após uma partida única sobre disfunção muscular e danos em jogadores de futebol.

Investigar a eficácia da imersão em água fria após o jogo de futebol

Testar a hipótese de que a massagem ajuda na recuperação muscular pósexercício, através do aumento do fluxo sanguíneo muscular para melhorar remoção de ácido láctico.
Crioimersão logo após um exercício extenuante reduziu o estresse oxidativo

Imersão em água fria foi eficaz, reduzindo alguns marcadores bioquímicos, funcionais e perceptivos de dano muscular.

A crioimersão diminuiu a percepção de dor nas pernas a fadiga geral nos jogadores

A massagem desportiva compromete 0 fluxo sanguíneo, impedindo a remoção de ácido láctico sanguíneo.

\section{DISCUSSAOO}

A presente revisão buscou verificar a eficácia da técnica de crioimersão e massagem desportiva na recuperação muscular de atletas pós-exercícios. Foram encontrados na literatura 15 artigos tratando de crioterapia pós-exercício.

Os principais achados neste estudo mostraram que a crioimersão diminuiu significativamente a percepção de dor e fadiga nos atletas. Hohenauer et al. ${ }^{13}$ constatou que a crioimersão minimizou consideravelmente os sintomas de DOMS 24, 48 e 96 horas após o exercício. Rowsell et al., ${ }^{14}$ em um estudo prévio verificou uma diminuição na percepção de dor nas pernas e fadiga geral em jogadores durante um torneiro de futebol, já Hayter ${ }^{15}$ et al. notou uma redução dos efeitos da fadiga muscular, ajudando na recuperação do desempenho máximo no ciclismo. Em uma recente revisão, a maioria dos estudos houve melhora no cansaço muscular, dor, bem-estar após exercícios extenuantes, capacidade indutiva anti-inflamatória da imersão em água fria ${ }^{16}$ e levando uma diminuição da temperatura da pele, podendo ser uma explicação para a diminuição da percepção de dor muscular. ${ }^{17}$

Yeung et al. ${ }^{17}$ observaram uma diminuição da frequência cardíaca, temperatura corpórea, aumento na disponibilidade de oxigênio muscular e aumento da capacidade 
oxidativa após imersão em água fria, resultados semelhantes foram observados no estudo de Roberts et al., ${ }^{18}$ onde denotaram uma diminuição da hemodinâmica cardíaca e temperatura tecidual no grupo de crioimersão, enquanto a recuperação ativa manteve a hemodinâmica e a temperatura pós exercício de resistência. Apoiando os achados citados, Prado et al. ${ }^{19}$ mostraram uma diminuição significativa do estresse oxidativo com a crioimersão, uma vez que esse estresse pode estar associado a fadiga, à redução da performance e ao dano muscular.

Dentre os artigos pesquisados o tempo mais comum de aplicação foi de 10 a 15 minutos, com a temperatura variando entre 10 a $15^{\circ} \mathrm{C}$, dados que coincidiram com o estudo de Machado et al., ${ }^{20}$ que as temperaturas entre 10 e $15^{\circ} \mathrm{C}$ com o tempo entre de 10 a 15 minutos podem proporcionar melhores resultados imediatos ou mais tardios.

Outra técnica de recuperação retratada nesta revisão foi a massagem desportiva, frequentemente utilizada pós-exercício. Nunes et $\mathrm{al}^{1}$ realizou um trabalho com 74 atletas, que completaram uma prova de Triatlhon de longa distância, dividiram dois grupos, um com massagem desportiva no quadríceps por 10 minutos e um grupo controle, houve uma diminuição na percepção de dor e fadiga no grupo experimental, porém não teve redução significativa na dor de pressão. Mesmo resultado relatado por Crane et al., ${ }^{21}$ onde 11 jovens foram submetidos a 10 minutos de massagem no quadríceps após exercício, constatando um benefício clínico por reduzir a inflamação e promoção da angiogênese mitocondrial. Conforme Resnick, ${ }^{22}$ a massagem após o exercício diminuiu a frequência cardíaca e promoveu um relaxamento durante a recuperação, considerando que a massagem foi mais eficaz que a recuperação passiva. Não corroborando com os resultados encontrados, Witshire et al. ${ }^{23}$ realizou um estudo com 12 indivíduos, comparando a massagem, recuperação ativa e passiva, pós $40 \%$ de contração isométrica máxima no antebraço e verificou que a massagem comprometeu o fluxo sanguíneo e não ocorreu remoção de ácido lático sanguíneo.

Delextra et al. ${ }^{24}$ compararam o efeito da massagem desportiva e crioimersão em 16 atletas de basquete, sendo 8 mulheres e 8 homens, após partidas oficiais. Constou que houve uma diminuição da fadiga geral e dor nas pernas nos dois grupos, as mulheres tiveram uma menor percepção de fadiga nas duas situações e a imersão em água fria promoveu menos dor que o grupo da massagem desportiva, e teve uma melhora no desempenho de salto logo após a intervenção. 
DOI: 10.18606/2318-1419/amazonia.sci.health.v7n3p74-83

Revista Amazônia: Science \& Health

2019, Vol. 7, № 3 .

ISSN: 2318-1419
Rocha LS. Nunes RD. Reis GR. Brito Junior EBL.

Eficácia da crioimersão e massagem desportiva na recuperação de atletas: uma revisão da literatura

\section{CONSIDERAÇOES FINAIS}

Através dos atuais resultados, imersão em água fria e a massagem desportiva pareceram ser técnicas bastante eficazes na recuperação de atletas após exercícios, diminuindo consideravelmente a percepção de dor e fadiga muscular. Sendo assim, essas duas estratégias de tratamento se tornam aliadas do Fisioterapeuta Esportivo na reabilitação do atleta, podendo prevenir e melhorar seu rendimento na modalidade praticada.

\section{REFERÉNCIAS}

1 Nunes GS, Bender PU, Menezes FS, Yamashitafuji I, Vargas VZ, Wageck B. Massage therapy decreases pain and perceived fatigue after long-distance Ironman triathlon: a randomised trial. Journal of physiotherapy. 2016; 62(2):83-87.

2 Nédélec M, McCall A, Carling C, Legall F, Berthoin S, Dupont G. Recovery in soccer. Sports Medicine. 2012;42(12):997-1015.

3 Junge A, Engebretsen L, Mountjoy ML, Alonso JM, Renström PA, Aubry MJ, Dvorak J. Sports injuries during the summer Olympic games 2008. The American journal of sports medicine. 2009;37(11):2165-2172.

4 Arena SS, Carazzato JG. Relation between medical clinical monitoring and the incidence of sports injuries in young athletes of São Paulo. Revista Brasileira de Medicina do Esporte. 2007;13(4):217-221.

5 Vaile J, Halson S, GrahamS. Recovery review: science vs. practice. J Aust Strength Cond. 2010;18(2):5-21.

6 Hoffman MD, Badowski N, Chin J, Stuempfle KJ. A randomized controlled trial of massage and pneumatic compression for ultramarathon recovery. Journal of orthopaedic \& sports physical therapy. 2016;46(5):320326.

7 Kovacs MS, Baker LB. Recovery interventions and strategies for improved tennis performance. British journal of sports medicine. 2014;48(1):18-21.

8 Montgomery PG, Pyne DB, Hopkins WG, Dorman JC, Cook K, Minahan CL. The effect of recovery strategies on physical performance and cumulative fatigue in competitive basketball. Journal of sports sciences. 2008;26(11):1135-1145.

9 Weerapong P, Hume PA, Kolt GS. The mechanisms of massage and effects on performance, muscle recovery and injury prevention. Sports medicine. 2005;35(3): 235-256.

10 Adamczyk JG, Krasowska I, Boguszewski D, Reaburn P. The use of thermal imaging to assess the effectiveness of ice massage and cold-water immersion as methods for supporting post-exercise recovery. Journal of Thermal Biology. 2016;60:20-25.

11 Hohenauer E, Clarys P, Baeyens JP, Clijsen R. The effect of local cryotherapy on subjective and objective recovery characteristics following an exhaustive jump protocol. Open access journal of sports medicine. 2016;7:89.

12 Leeder JD, Van Someren KA, Bell PG, Spence JR, Jewell AP, Gaze D et al. Effects of seated and standing cold water immersion on recovery from repeated sprinting. Journal of sports sciences. 2015;33(15):1544-1552.

13 Hohenauer E, Taeymans J, Baeyens JP, Clarys P, Clijsen R. The effect of post-exercise cryotherapy on recovery characteristics: a systematic review and meta-analysis. PloS one. 2015;10(9):e0139028. 
DOI: 10.18606/2318-1419/amazonia.sci.health.v7n3p74-83

Revista Amazônia: Science \& Health

2019, Vol. 7, № 3 .

ISSN: 2318-1419
Rocha LS. Nunes RD. Reis GR. Brito Junior EBL.

Eficácia da crioimersão e massagem desportiva na recuperação de atletas: uma revisão da literatura

14 Rowsell GJ, Coutts AJ, Reaburn P, Hill-Haas S. Effect of post-match cold-water immersion on subsequent match running performance in junior soccer players during tournament play. Journal of sports sciences. $2011 ; 29(1): 1-6$.

15 Hayter KJ, Doma K, Schumann M, Deakin GB. The comparison of cold-water immersion and cold air therapy on maximal cycling performance and recovery markers following strength exercises. PeerJ. 2016;4:1841.

16 Lombardi G, Ziemann E, Banfi G. Whole-body cryotherapy in athletes: from therapy to stimulation. An updated review of the literature. Frontiers in Physiology. 2017;8:258.

17 Yeung SS, Ting KH, Hon M, Fung NY, Choi MM, Cheng JC et al. Effects of Cold Water Immersion on Muscle Oxygenation During Repeated Bouts of Fatiguing Exercise: A Randomized Controlled Study. Medicine. 2016;95(1):2455.

18 Roberts LA, Muthalib M, Stanley J, Lichtwark G, Nosaka K, Coombes JS, et al. Effects of cold water immersion and active recovery on hemodynamics and recovery of muscle strength following resistance exercise. American Journal of Physiology-Regulatory, Integrative and Comparative Physiology. 2015;309(4):389-398.

19 Prado FP, Paludetto DRB, Bachur CAK, Freitas RAL, Zaia JE, Neto TLB, et al. Estresse oxidativo no plasma sanguíneo de indivíduos submetidos ao esforço físico agudo seguido de crioimersão corporal. Fisioterapia e Pesquisa. 2012;19(3):215-221.

20 Machado AF, Ferreira PH, Micheletti JK, Almeida AC, Lemes ÍR, Vanderlei FM, et al. Can water temperature and immersion time influence the effect of cold water immersion on muscle soreness? $A$ systematic review and meta-analysis. Sports Medicine. 2016;46(4):503-514.

21 Crane JD, Ogborn DI, Cupido C, Melov S, Hubbard A, Bourgeois JM, et al. Massage therapy attenuates inflammatory signaling after exercise-induced muscle damage. Science translational medicine. 2012;4(119):119ra13-119ra13.

22 Resnick PB. Comparing the Effects of Rest and Massage on Return to Homeostasis Following Submaximal Aerobic Exercise: a Case Study. International journal of therapeutic massage \& bodywork. 2016;9(1):4.

23 Wiltshire EV, Poitras V, Pak M, Hong T, Rayner J, Tschakovsky ME. Massage impairs postexercise muscle blood flow and "lactic acid" removal. Med Sci Sports Exerc. 2010; 42(6):1062-71.

24 Delextrat A, Calleja-González J, Hippocrate A, Clarke ND. Effects of sports massage and intermittent coldwater immersion on recovery from matches by basketball players. Journal of sports sciences. 2013;31(1):1119.

25 Tiidus PM. Alternative treatments for muscle injury: massage, cryotherapy, and hyperbaric oxygen. Current reviews in musculoskeletal medicine. 2015;8(2):162-167.

26 Bervoets DC, Luijsterburg PA, Alessie JJ, Buijs MJ, Verhagen, AP. Massage therapy has short-term benefits for people with common musculoskeletal disorders compared to no treatment: a systematic review. Journal of physiotherapy. 2015;61(3):106-116.

27 Crowther F, Sealey R, Crowe M, Edwards A, Halson S. Team sport athletes' perceptions and use of recovery strategies: a mixed-methods survey study. BMC Sports Science, Medicine and Rehabilitation. 2017;9(1):6.

28 Pointon M, Duffield R. Cold water immersion recovery after simulated collision sport exercise. Medicine and science in sports and exercise. 2012;44(2):206-216.

29 Ascensao A, Leite M, Rebelo AN, Magalhães S, Magalhães J. Effects of cold water immersion on the recovery of physical performance and muscle damage following a one-off soccer match. Journal of sports sciences. 2011;29 (3):217-225. 
DOI: 10.18606/2318-1419/amazonia.sci.health.v7n3p74-83

Revista Amazônia: Science \& Health

2019, Vol. 7, № 3 .

ISSN: 2318-1419
Rocha LS. Nunes RD. Reis GR. Brito Junior EBL.

Eficácia da crioimersão e massagem desportiva na recuperação de atletas: uma revisão da literatura

30 Versey NG, Halson SL, Dawson, BT. Water immersion recovery for athletes: effect on exercise performance and practical recommendations. Sports medicine. 2013;43(11):1101-1130. 